\title{
Fundamental Studies of Vortices Induced by a Vortex-generator for Automotive Applications
}

\author{
Asiful Islam ${ }^{1, a}$, Dr. Graham Doig ${ }^{2, b}$ \\ ${ }^{1,2}$ UNSW School Of Mechanical \& Manufacturing Engineering, Sydney Australia \\ aasiful@unsw.edu.au, bg.doig@unsw.edu.au \\ *Asiful Islam, asiful@unsw.edu.au, +614 31863553
}

\begin{abstract}
Keywords: Vane-type, vortex-generator, boundary layer, separation, core drift, automotive aerodynamics
\end{abstract}

\begin{abstract}
For automotive applications, passive flow control devices can be used to reduce, delay or prevent flow separation. This study explores the nature of vortex generation and behaviour, numerically and experimentally, for a simple geometry at a Reynolds Number $\left(\operatorname{Re}_{\mathrm{x}}\right)$ of $5 \times 10^{5}$ and $1.945 \times 10^{6}$. The setup comprised a triangular vane vortex-generator mounted on a shallow ramp referenced from literature. Flow over the isolated ramp was validated with past experimental particle-image-velocimetry (PIV) data, which also highlighted the relative performance of various turbulence models. A parametric study was undertaken with the vane orientation defined by an angle-of-attack $(\beta)$ and stream-wise location $\left(\mathrm{x}_{\text {edge }} / \mathrm{x}_{\mathrm{VG}}\right)$. These results revealed relationships between geometric parameters of the vortex generator, as well as the influence of the boundary layer thickness $\left(\mathrm{h}_{\mathrm{VG}} / \delta\right)$, on the spatial trajectory of induced vortices.
\end{abstract}

\section{Introduction}

Most modern formula racing cars include fins, barge boards, turning vanes and vortex generators (VGs) to control the airflow. Flow separation generally occurs due to the momentum loss in the boundary layer as it acts against an adverse pressure gradient [1], [2]. Typical race cars are designed for high downforce, but the large gradients associated with this are separation-prone. Vortices are also often generated in order to influence specific components elsewhere on the vehicle, which can be far downstream and therefore sensitive to vortex path variation and decay behaviour.

The present work explores the influence of VG position and orientation on the trajectory of induced vortices emanating from a simple vane-type configuration. Although previous studies have identified some of the generic features [2] of vortex generators, further progress is required in characterizing the downstream core behaviour and assessing the suitability or otherwise of steadystate Reynolds-Averaged Navier-Stokes (RANS) modeling (typical in the motorsport industry) for this application.

The vane, in both experiments and simulations, was positioned on a ramp geometry which provided some complexity to the baseline flow and pressure distribution as a loose proxy for a realworld scenario. The ramp was inspired by Beves [1], who studied flow control using surface dimples and provided a good starting point for validation. Initially, the ramp geometry was simulated as a valid and verified baseline prior to application of flow control. Secondly, the VGs were attached to the ramp and simulated under identical free-stream flow at different orientations. Latter set of simulations also incorporated higher velocities and was supported by wind tunnel experiments.

\section{Methodology}

A triangular-shaped, $26.57^{\circ}$ vane-type $\mathrm{VG}, 30 \mathrm{~mm}$ long $\left(\mathrm{x}_{\mathrm{VG}}\right)$ and $15 \mathrm{~mm}$ high $\left(\mathrm{h}_{\mathrm{VG}}\right)$ was used throughout the present study. This had an aspect ratio of $2\left(\mathrm{x}_{\mathrm{VG}} / \mathrm{h}_{\mathrm{VG}}\right)$ and was modelled to represent a $2 \mathrm{~mm}$ thick plate. Dimensions of the wind tunnel test section (Fig. 1) used by Beves [1] was numerically replicated, with an upstream ramp angle of $5.5^{\circ}$ and a downstream ramp angle of $16^{\circ}$. 
His PIV measurements were taken at three inspection planes X50, X100 and X200 (Fig. 1). A nearidentical meshing strategy was used with the VG-inclusive domain, constructed after this initial validation stage and these results were experimentally validated after collaboration with RMIT University. For the sake of brevity, this will only be briefly mentioned, with more emphasis on validation against the original ramp flow.

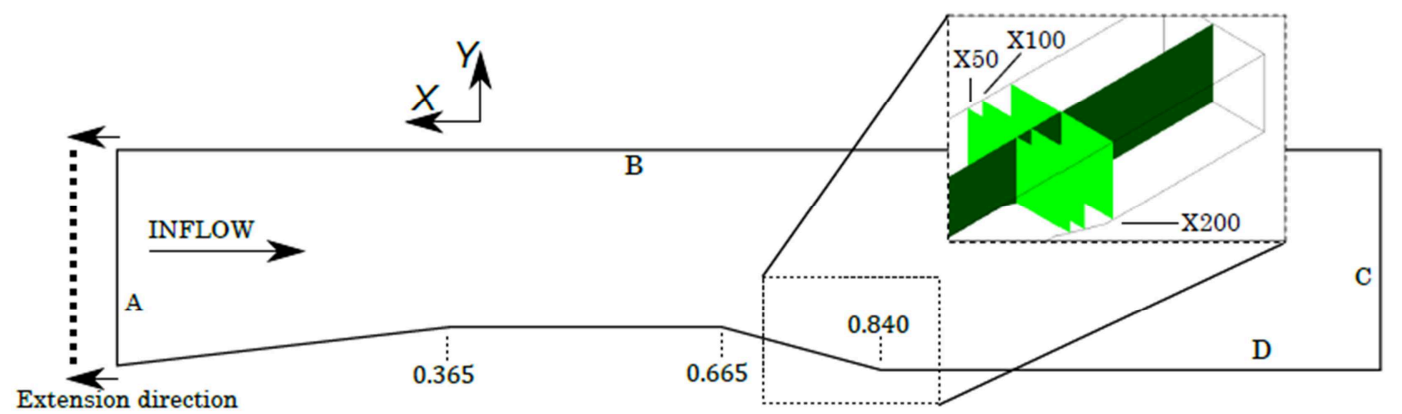

Figure 1: Original layout of the $350 \mathrm{~mm}$ wide wind tunnel test section defined by Beves [1] marked with the primary longitudinal and cross-stream inspection planes used in this study.

Table 1: Boundary conditions defined for the reference flow domain shown in Fig 1.

\begin{tabular}{cccc}
\hline A & B & C & D \\
\hline $4.5 \mathrm{~m} / \mathrm{s}$ inlet & Zero-shear wall & 0 Pa Pressure Outlet & No-Slip wall \\
\hline
\end{tabular}

Wind-tunnel experiments at RMIT utilised four-hole Cobra probes with a 13-second sampling time to resolve all three velocity components downstream of the VG. A $5 \times 4$ measurement grid was used for different angles-of-attack, at 1 VG-length downstream of the tail.

\section{Numerical Model}

A 64-bit commercial pressure-based solver, ANSYS Fluent, was used with second order upwinding for discretisation. The isolated ramp domain was examined with 4 different fullystructured, hexahedral meshes. The local boundary layer was captured by approximately 20 and 24 cells for the reference and fine mesh respectively. All the final meshes achieved $0.6<y^{+}<3$ along the ramp. This ensured that wall functions were not required [3]. Turbulence properties at the inlet matched measurements where available.

The selected mesh for the isolated ramp consisted of 3.002 million cells and a convergence study was undertaken using non-uniform grid-convergence-index (GCI) [4]. An average edge length ratio of $\mathrm{r}_{\mathrm{i}} \approx 1.2$ was used in all coordinate directions. GCI calculations indicated maximum errors bounds of $17.8 \%$ using $C_{p}$ values. Further refinement to a 4.176 million cell mesh would only lead to a $4.63 \%$ reduction in predicted ramp drag $F_{d}$.

A separate grid-convergence study for the VG-inclusive domain used 4 different levels of refinement (Fig. 2). A 3.352 million cell mesh was finally selected. Further refinement of this mesh would only lead to $0.03 \%$ change in the VG drag force (Fig. 2). The mesh choice maximised computational efficiency with minimal influence on results.

VG-inclusive transient simulations used an optimal time step of $\Delta t=0.0005$ seconds with the Courant number $\leq 1$ to satisfy the CFL criteria. All simulations reached a steady state after two flow cycles, mainly due to the diffusivity of Reynolds-Averaged Navier-Stokes (RANS) models. All normalized transport residuals fell below $10^{-6}$ prior to collection of results. Convergence of the flow field was also monitored using a point $50 \mathrm{~mm}$ off-the-wall at the bottom of the ramp.

Boundary layer thickness is an important factor for the performance of a vortex-generator and implementation of the correct vane size. This also controls the $\mathrm{h}_{\mathrm{VG}} / \delta$ ratio, which has an influence on vortex trajectories shown in the results which follow. A fully developed boundary layer was established ahead of the ramp using a $1.717 \mathrm{~m}$ extension (Fig. 1), giving $\operatorname{Re}_{\mathrm{x}}=5 \times 10^{5}$ from flat plate approximations [3]. 


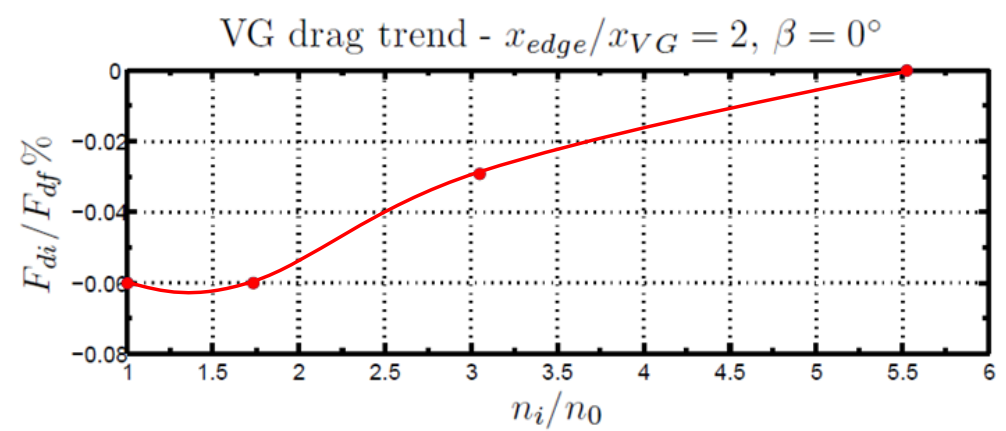

Figure 2: Grid convergence with $60 \mathrm{~mm}$ VG position for $\beta=0^{\circ}$. The $y$-axis $\%$ change was normalised by finest mesh $F_{d}$. The $x$-axis has each mesh size $\left(n_{i}\right)$ normalised by the coarsest mesh $\left(n_{0}\right)$.

Turbulence Modelling. Results were obtained using the Shear Stress Transport (SST) k- $\omega$ model [5] [6]. This proved to be the most robust and accurate after comparative studies against PIV data (Fig. 3). Wilcox's low-Re k- $\omega$ model, Realizable k- $\varepsilon$ and the Shear Stress Transport (SST) k- $\omega$ were all compared in reaching this conclusion. For brevity, one PIV comparison is shown, but it is representative of other results obtained on all 3 inspection planes (Fig. 1) from Beves [1]. Velocity profiles at $50 \mathrm{~mm}$ after the trailing edge highlight that close to the surface, the low-Re k- $\omega$ model overpredicted the recirculation region. An $\omega$-based Reynolds Stress Model was omitted from further comparisons due to lack of clear convergence and computational effort.

The SST k- $\omega$ model was the best at matching the major trends in the flow profile. Although, the zonal approach includes an improved blending function [6], the transition between the viscous region and free-stream $(45<\mathrm{y}<40)$ still requires improvement (Fig. 3). Overall, given their computational efficiency, a RANS approach can still be used to model induced vortices however; it should only be used after validating at a specific Reynolds Number and turbulence intensity.
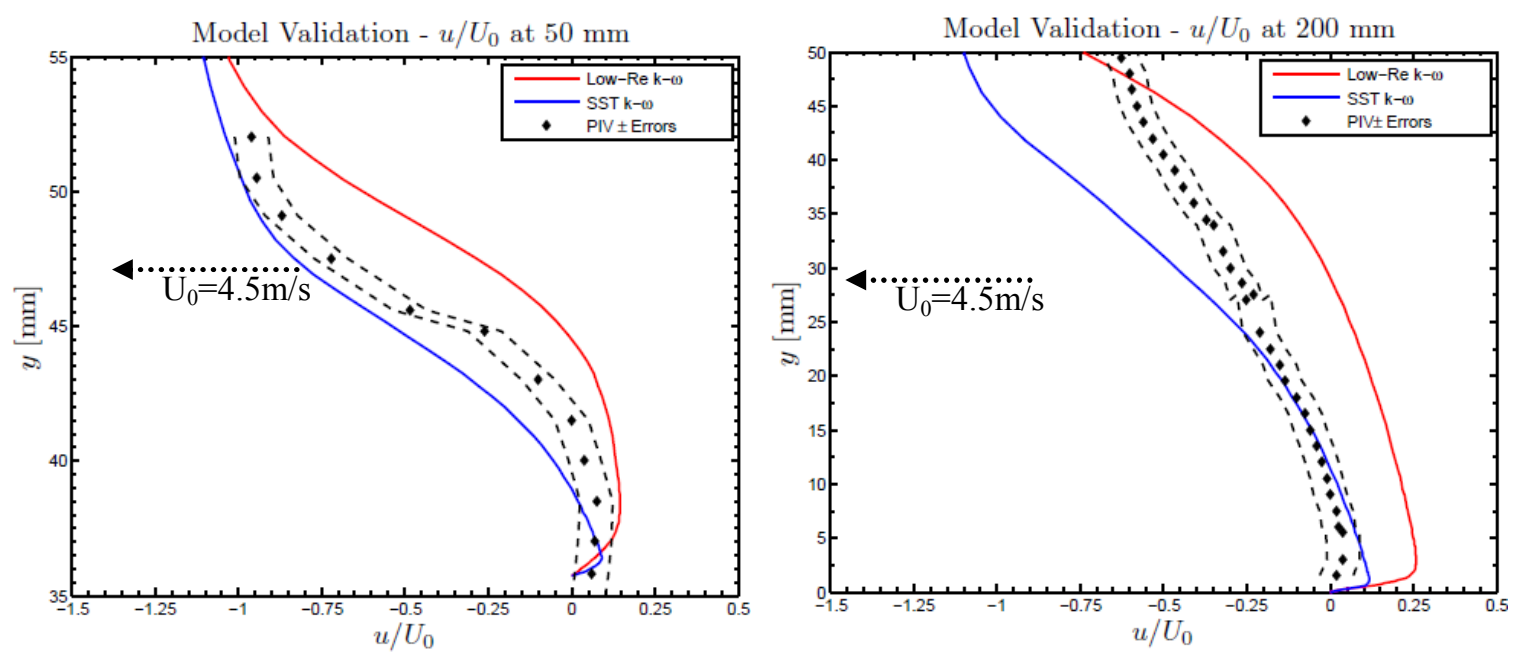

Figure 3: Comparison of CFD results against existing PIV data at $50 \mathrm{~mm}$ (left) and $200 \mathrm{~mm}$ (right) downstream of the ramp's trailing edge.

\section{Results \& Discussion}

Vortex influence. Vortices were induced by vanes oriented at $0^{\circ}, 5^{\circ}, 10^{\circ}$ and $15^{\circ}$ (Fig. 4 ) to the free-stream. Triangular vanes were selected primarily due to the single point of vortex initiation and their popularity arising from relatively low drag penalty [2]. Aerodynamic drag is commonly used as a performance indicator and total drag on the ramp and VGs were both monitored here. 

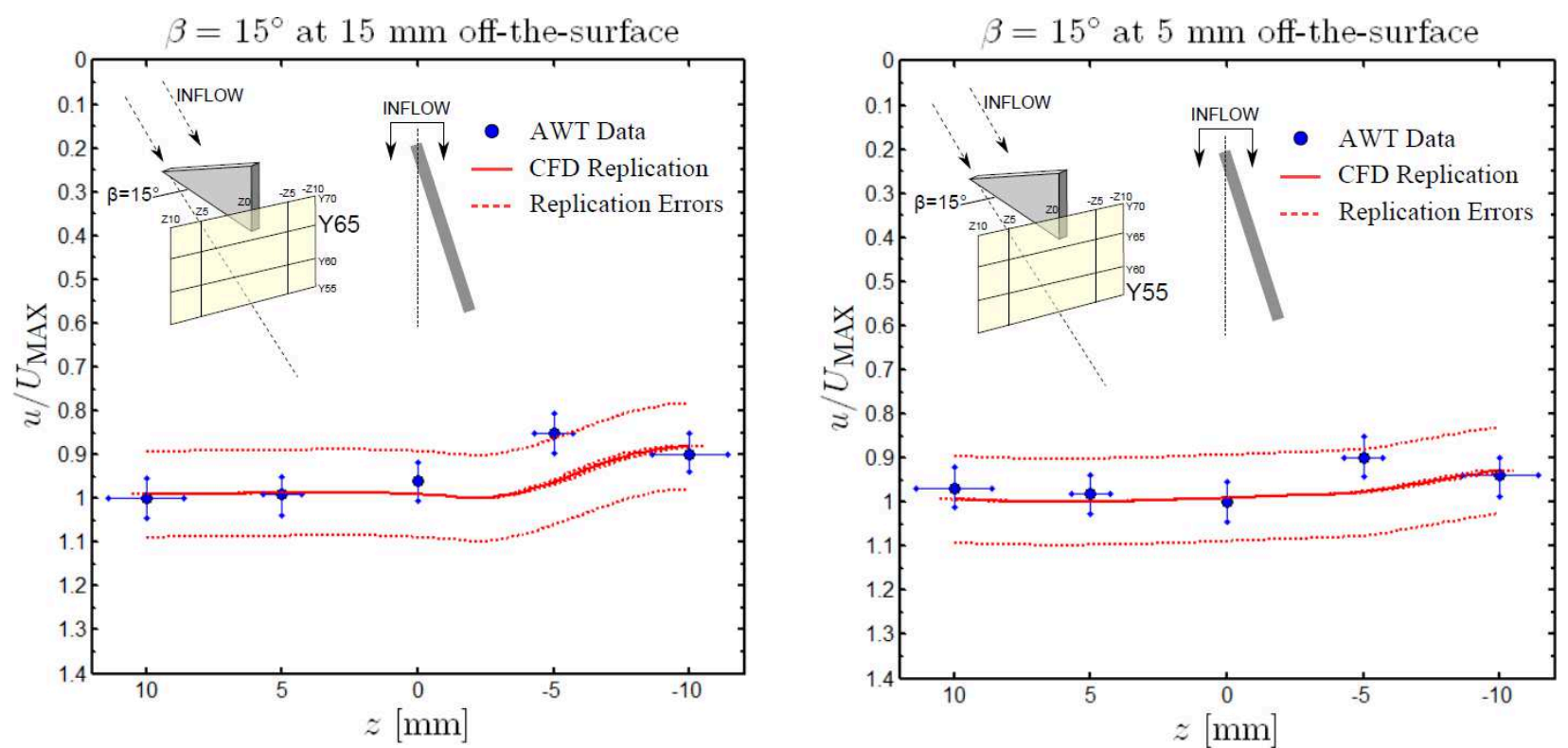

Figure 4: Wind-tunnel data for $17.5 \mathrm{~m} / \mathrm{s}$ and CFD validation model for $60 \mathrm{~mm}$ position with $\beta=15^{\circ}$.

At the $60 \mathrm{~mm}$ position, changing VG orientation from $\beta=0^{\circ}$ to $5^{\circ}$ increased the total ramp drag by $5.7 \%$, from 0.1085 to $0.1147 \mathrm{~N}$. However, the drag sensitivity is reduced at higher angles-ofattack $(\beta)$. This is highlighted by a $0.04 \%$ drag decrease as the vane is altered from $10^{\circ}$ to $15^{\circ}$. This reveals the importance of the interaction between induced vortices and coherent structures in the boundary layer, since the total ramp drag decreases despite an increase in the isolated VG drag.

The VG drag force was greater for $240 \mathrm{~mm}$ compared to the $60 \mathrm{~mm}$ position, for all angles-ofattack. For $\beta=15^{\circ}$, there was a $2.8 \%$ increase in drag force corresponding to position change. VGs at this position also had a greater impact on the flow and the total ramp drag actually decreased by $2.2 \%$, on average, relative to the $60 \mathrm{~mm}$ position.

Vortex development. Based on excellent correlation with wind-tunnel measurements (Fig. 4), the CFD model was deemed to be accurately capturing vortex behaviour. Using velocity contours on cross-stream planes, two distinct flow features were observed immediately after the VGinteraction. Acting as an obstruction to the flow, these vanes induce a low-pressure wake. This leads to a discernable yet short-lived vortex, especially prominent for higher $\beta$ values.

In addition to this, the vanes also form a primary longitudinal vortex which increased localised vorticity. This was observed as a VG augmenting the rotational potential of the flow in that region. From time-averaged data, a stream-wise velocity deficit in the core was identified (Fig. 4) and this was stable in space for all $\beta$.

Initially, the vortex strength was directly proportional to the angle-of-attack $\beta$. C-S Yao and J. C. Lin [7] also found that circulation $\left(\Gamma_{\mathrm{P}}\right)$ increased with the angle-of-attack and $(\sin \beta)^{1.5}$ represented functional relationship near their rectangular VG.

In this project, an adverse-pressure gradient established by the backward-facing ramp (Fig. 1), distorted the induced vortices. This is consistent with past observations by Simpson [8] in his study of turbulent boundary layers. This project also revealed that skewness of the primary vortex was proportional to $\beta$.

From conservation of angular momentum, greater vortex distortion and skewness, also contributes to reduced circulation, as the core radius increases. From comparative studies of low and conventional VGs, Yao and Lin [7] also identified an elliptical rather than circular profile for the embedded vortices near the wall, confirming the observations in this study.

Vortex core trajectory. Range of $\beta$ values used here was governed by literature, since Yao and Lin [7] identified reduced effectiveness for $\beta>16^{\circ}$ with rectangular vanes. Various methods for vortex detection was explored however a local vorticity maxima $(\omega=\nabla \times \mathbf{V})$ was found to be the most reliable. Downstream inspection planes were placed at $10 \mathrm{~mm}$ increments from the tail and normalised by vane length $\left(\mathrm{x}_{\mathrm{VG}}\right)$. The tracks in the vertical, wall- 
normal direction were easier to locate than the lateral direction and might be related to mesh density and growth rates. As the cell sizes grow larger, the vortex core was more difficult to identify and hence, some unrealistic deviations were observed in z-direction, especially downstream of the ramp.
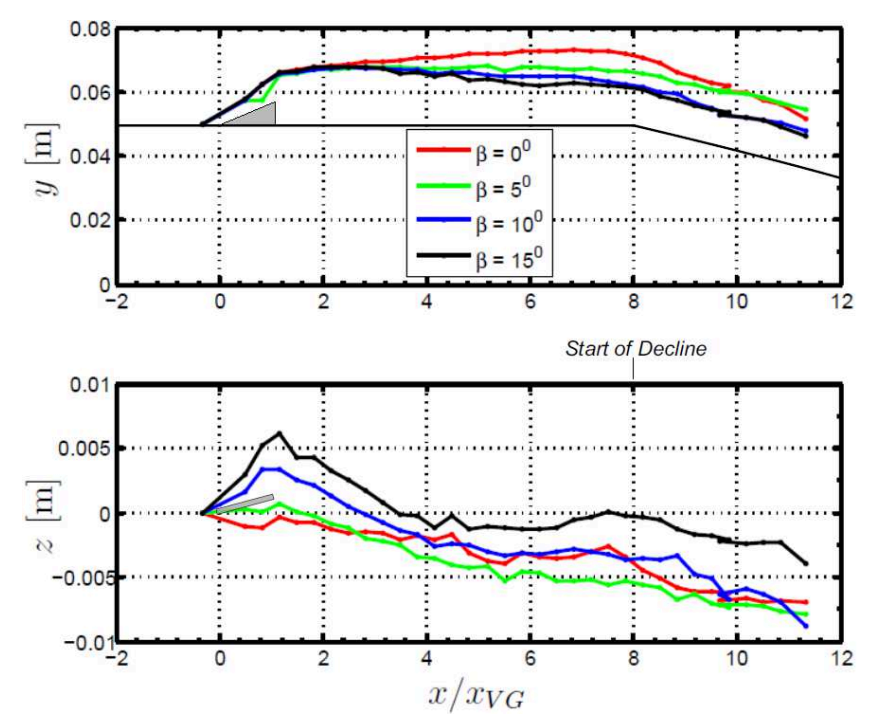

Figure 5: Vertical (Y) and lateral (Z) vortex core drift for the VG located at $60 \mathrm{~mm}$ off the leading edge.

For the $60 \mathrm{~mm}$ position, 38 inspection planes were required, as shown in Fig. 5. As it approaches the adverse pressure gradient the core became larger, with increased skewness. For the $60 \mathrm{~mm}$ position (Fig. 5) the primary vortex rose away from the wall at a high gradient for approximately $1.167 \mathrm{x}_{\mathrm{VG}}$ distance from the tip. As the size of the core increased and interacted with the free-stream, the vortices reached a terminal height, with the $0^{\circ}$ orientation having a maximum $1.52 \mathrm{~h}_{\mathrm{VG}}$ elevation.

The vortices reach a stable height and only vary between $0.053 \mathrm{~h}_{\mathrm{VG}}$ and $1.52 \mathrm{~h}_{\mathrm{VG}}$ for all $\beta$ within $1<\mathrm{x} / \mathrm{x}_{\mathrm{VG}}<7.8$ downstream of the tip (Fig 5). For $\beta=10^{\circ}$ and $15^{\circ}$ the increased vortex strength also corresponds to a rapid core descent towards the wall. The pressure gradient also influences the vortex core trajectories and all the induced vortex trajectories tend to follow the $16^{\circ}$ ramp beyond $\mathrm{x}$ $=8 \mathrm{x}_{\mathrm{VG}}$ and $\mathrm{x}=2 \mathrm{x}_{\mathrm{VG}}$ for $60 \mathrm{~mm}$ and $240 \mathrm{~mm}$ charts, respectively. This was followed by rapid diffusion of the vortices.

Boundary layer separation also had an influence on the primary and secondary vortices. This led to the above mentioned diffusion and the change in both the $\mathrm{Y}$ and $\mathrm{Z}$ gradients. For $\beta=0^{\circ}$ the natural trajectory over the tip led to maximum elevation from the surface relative to the other angles. However, they generated stronger vortices with lower trajectories over the surface.

The $240 \mathrm{~mm}$ location resulted in much greater lateral vortex drift (Fig. 6) and this also encountered the thickest boundary layer leading to $h_{\mathrm{VG}} / \delta=0.410$. The vortices induced from the vane at $240 \mathrm{~mm}$ position, had a lower profile and elevation from the surface, which might be a contributor to the lower overall drag mentioned.

When VGs are placed on curved, external surfaces with an adverse gradient, they induce vortices which interact with the surface. Although this assists in reduction of total drag, the vortices are highly skewed and have greater levels of interaction with the wall, leading to relatively rapid decay. Hence, a compromise needs to be made between using such vortices for aerodynamic effects far downstream or to simply achieve a minimal drag design. 


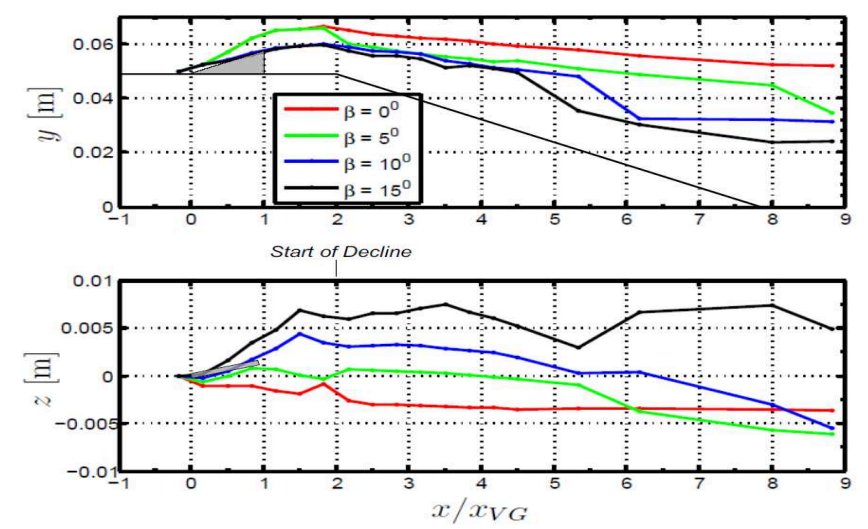

Figure 6: Vertical (Y) and lateral (Z) vortex core drift for the VG located at $240 \mathrm{~mm}$ off the leading edge.

\section{Summary}

Isolated, triangular vanes were mounted ahead of a $16^{\circ}$ decline at 60 and $240 \mathrm{~mm}$ positions from a leading edge, to understand vortex generation and drift. The $\mathrm{h}_{\mathrm{VG}} / \delta$ was varied and the vanes augmented the vorticity in the boundary layer. Skewed vortices were also formed due to the adverse pressure gradient, leading to rapid diffusion.

VG position on an external surface $\operatorname{affectsh}_{\mathrm{VG}} / \delta$, significantly altering the downstream vortex behaviour. From the $60 \mathrm{~mm}$ position, vortex proximity to the surface was proportional to $\beta$. Larger angles led to vortex trajectories closer to the wall. The $\mathrm{h} / \delta$ ratio at $60 \mathrm{~mm}$ location was better at inducing the flow to follow the surface curvature. The $240 \mathrm{~mm}$ position was closest to the trailing edge and was effective at reducing overall drag while providing improved control in the span-wise direction. The $10^{\circ}$ and $15^{\circ}$ orientations at the $240 \mathrm{~mm}$ location should be capable of diverting flow around adjacent components and increase localised velocity and vorticity downstream.

The VG orientation, position and influence of the pressure gradient were all used to gain an insight into the spatial trends of induced vortices. In the near-future, with more realistic upstream disturbances and velocities, this project will assist the placement of VGs for various automotive surfaces such as on side pods of formula racing cars. Vortex decay and surface interactions are currently being studied further.

\section{References}

[1] C. C. Beves, "An Investigation into the use of low aspect ratio spherical wells to reduce the flow separation on an inverted wing in ground effect," The University Of New South Wales, Sydney, Australia, 2009.

[2] C. M. Velte, "Characterisation of Vortex Generator Induced Flow," Department of Mechanical Engineering, Technical University of Denmark, Lyngby, Denmark, July, 2009.

[3] H. K. Versteeg and W. Malalasekera, An Introduction to Computational Fluid Dynamics - The Finite Volume Method 2nd Edition, Essex: Pearson - Prentice Hall, 2007.

[4] P. J. Roache, "Perspective: A Method for Uniform Reporting of Grid Refinement Studies," Journal of Fluids Engineering, vol. 116, pp. 405-413, September, 1994.

[6] F. R. Menter, M. Kuntz and R. Langtry, "Ten Years of Industrial Experience with the SST Turbulence Model," in Turbulence, Mass and Heat Transfer IV, Antalya, Turkey, 12-17 October 2003.

[7] C. S. Yao, J. C. Lin and B. G. Allan, "Flow-Field Measurement of Device-Induced Embedded Streamwise Vortex on a Flat Plate," in 1st AIAA Flow Control Conference, St. Louis, MO, 2427 June, 2002.

[8] R. L. Simpson, "Aspects of turbulent boundary-layer separation," Progress in Aerospace Sciences, vol. 32, no. 5, pp. 457-521, 1996. 
Copyright of Applied Mechanics \& Materials is the property of Trans Tech Publications, Ltd and its content may not be copied or emailed to multiple sites or posted to a listserv without the copyright holder's express written permission. However, users may print, download, or email articles for individual use. 